\title{
Optimal biological dose: a systematic review in cancer phase I clinical trials
}

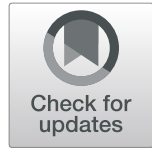

J. Fraisse ${ }^{1+}$, D. Dinart ${ }^{2+}$, D. Tosi ${ }^{1}$, C. Bellera ${ }^{2}$ and C. Mollevi ${ }^{1,3^{*}}$ (D)

\begin{abstract}
Background: Classical phase 1 dose-finding designs based on a single toxicity endpoint to assess the maximum tolerated dose were initially developed in the context of cytotoxic drugs. With the emergence of molecular targeted agents and immunotherapies, the concept of optimal biological dose (OBD) was subsequently introduced to account for efficacy in addition to toxicity. The objective was therefore to provide an overview of published phase 1 cancer clinical trials relying on the concept of OBD.

Methods: We performed a systematic review through a computerized search of the MEDLINE database to identify early phase cancer clinical trials that relied on OBD. Relevant publications were selected based on a two-step process by two independent readers. Relevant information (phase, type of therapeutic agents, objectives, endpoints and dose-finding design) were collected.
\end{abstract}

Results: We retrieved 37 articles. OBD was clearly mentioned as a trial objective (primary or secondary) for 22 articles and was traditionally defined as the smallest dose maximizing an efficacy criterion such as biological target: biological response, immune cells count for immunotherapies, or biological cell count for targeted therapies. Most trials considered a binary toxicity endpoint defined in terms of the proportion of patients who experienced a doselimiting toxicity. Only two articles relied on an adaptive dose escalation design.

Conclusions: In practice, OBD should be a primary objective for the assessment of the recommended phase 2 dose (RP2D) for a targeted therapy or immunotherapy phase I cancer trial. Dose escalation designs have to be adapted accordingly to account for both efficacy and toxicity.

Keywords: Phase 1 clinical trial, Cancer, Dose finding study, Optimal biological dose

\section{Background}

The primary objective of phase 1 cancer clinical trials is to assess the maximum tolerated dose (MTD) based on the dose limiting toxicity (DLT) evaluated in most of the cases on the first cycle of treatment, the safety profile and the recommended phase 2 dose (RP2D) [1]. Most dose-finding designs available for phase 1 cancer clinical trials were

\footnotetext{
* Correspondence: Caroline.Mollevi@icm.unicancer.fr; https://orcid.org/00000003-4827-3684

†. Fraisse and D. Dinart contributed equally to this work.

${ }^{1}$ Unité de Biométrie, Institut du Cancer Montpellier (ICM), Université de Montpellier, 208 rue des Apothicaire, 34298 Montpellier Cedex 5, France ${ }^{3}$ Institut Desbrest d'Epidémiologie et de Santé Publique, UMR Inserm Université de Montpellier, Montpellier, France

Full list of author information is available at the end of the article
}

initially developed in the context of cytotoxic conventional agents. These methods are based on an underlying hypothesis which implies that the dose of a cytotoxic drug is related to the toxic response via an increasing monotonic relationship [2]. With the emergence of molecular targeted agents and immunotherapies and given their specific mechanism of action, this paradigm has been modified. Severe toxicities are rare, often delayed in subsequent treatment cycles, preventing the MTD from being reached [3]. As such, dose-finding designs based only on a toxicity endpoint may not be appropriate anymore. In this context, the concept of optimal biological dose (OBD) has been introduced, which accounts for efficacy in addition to toxicity.

(c) The Author(s). 2021 Open Access This article is licensed under a Creative Commons Attribution 4.0 International License, which permits use, sharing, adaptation, distribution and reproduction in any medium or format, as long as you give appropriate credit to the original author(s) and the source, provide a link to the Creative Commons licence, and indicate if changes were made. The images or other third party material in this article are included in the article's Creative Commons licence, unless indicated otherwise in a credit line to the material. If material is not included in the article's Creative Commons licence and your intended use is not permitted by statutory regulation or exceeds the permitted use, you will need to obtain permission directly from the copyright holder. To view a copy of this licence, visit http://creativecommons.org/licenses/by/4.0/ The Creative Commons Public Domain Dedication waiver (http://creativecommons.org/publicdomain/zero/1.0/) applies to the data made available in this article, unless otherwise stated in a credit line to the data. 
Assessing the OBD instead of the classical MTD thus appears particularly relevant for modern phase I trials [4].

OBD is generally defined as the lowest dose providing the highest rate of efficacy while being safely administered. To our knowledge, there is however no consensus on the efficacy endpoint to be accounted for in the OBD, nor on the most appropriate dose escalation strategy to apply when assessing OBD.

Several efficacy endpoints and dose escalation designs have been proposed in the context of OBD, as it requires to simultaneously account for efficacy and toxicity. With regards to dose-escalation designs, Piantadosi and Liu proposed a variant of the continual reassessment method (CRM) dose-escalation design [5], which models the dose-efficacy curve via an auxiliary pharmacokinetics (PK) measurement (area under the curve, AUC) using a two-parameter logistic dose-efficacy model [6]. Braun proposed also to extend the CRM to a bivariate trial design for two competing outcomes: toxicity and disease progression [7]. Bekele and Shen proposed a bayesian approach to jointly model the dose-toxicity and doseefficacy curves [8]. The authors expressed toxicity using a binary variable (presence or absence of toxicity), while efficacy was modeled using a continuous biomarker expressing the concentration of a target protein. This sequential method specifically models the correlation between toxicity and efficacy via a latent Gaussian variable. Dragalin and Fedorov proposed a similar method where patient response is characterized by two dependent binary outcomes, one for efficacy and one for toxicity, using either a bivariate logistic model or a Cox bivariate binary model $[9,10]$. Houede et al. proposed an outcome-adaptive bayesian design with toxicity and efficacy characterized by ordinal variables, with efficacy defined as complete response, partial response, stable disease or progressive disease, and toxicity defined as a three-level ordinal variable representing the worst severity of adverse events [11]. Individual probabilities of severe toxicity and tumor response are then sequentially jointly re-estimated.

Overall, these developments highlight the heterogeneity in terms of both efficacy endpoints and dose escalation designs in the context of OBD. Efficacy may rely on pharmacokinetic or pharmacodynamic (PD) endpoints, clinical or radiological measures, or biomarkers such as immune response. Similarly, methodological developments have led to various phase I designs (bivariate models vs joint models, binary vs ordinal variables, etc.). The development of novel therapeutic anti-cancer agents has challenged traditional approaches conducting phase 1 trials. The objective of the present work was therefore to provide an overview of recent phase 1 cancer clinical trials relying on the concept of $\mathrm{OBD}$, with a particular focus on (i) how efficacy is accounted for in the definition of OBD, and (ii) dose-escalation designs allowing for the estimation of OBD.

\section{Methods}

\section{Selection}

The systematic review involved two steps: selection of relevant manuscripts and data extraction. We performed a systematic review through a computerized search of the MEDLINE database to identify cancer early phase clinical trials that relied on OBD. The search algorithm was the following: ((((“"Optimal" AND ("Biologic" OR "Biological") AND "Dose") AND "cancer"[Filter]) AND "humans"[Filter]) AND (“2000/01/01”[Date - MeSH]: “2019/12/31”[Date $\mathrm{MeSH}]))$ ) AND "clinical trial"[Filter]. We selected relevant publications based on a two-step process using a standardized data extraction grid designed and validated by two readers who independently checked both steps of the selection process. Discrepancies were resolved by mutual consensus. In the first step, general information was retrieved based on the abstract. Publications were ineligible if the abstract presented at least one of the following characteristics: letter/comment to the editor; conference abstract; not conducted in humans; not related to cancer; not a phase I trial. In the second step of the selection process, we read the full manuscripts of the selected abstracts. Publications were ineligible if they included at least one of the following characteristics: absence of an efficacy endpoint, absence of a toxicity endpoint; methodological paper. Results of the selection process are presented following the PRISMA guidelines for the reporting of systematic reviews and meta-analyses $[12,13]$.

\section{Data collection and analysis}

For full manuscripts that met eligibility criteria, we collected information regarding general characteristics of the articles: title of the article, phase of the study, localization of the cancer, molecules tested (single or association), type of therapeutic agents, principal and secondary objectives, primary and secondary endpoints (among toxicity and efficacy) and dose-finding design. We collected definitions for the OBD, MTD, DLT, as well as observation period for assessment of the DLT.

We provide a descriptive analysis of the publications. Quantitative variables were reported using descriptive statistics (median, minimum and maximum). For qualitative variables, we provided counts $(\mathrm{N})$ and proportions (\%) of each modality. Analyses were performed using the STATA software (version 16; STATA, College Station, TX). 


\section{Results}

\section{Trial selection}

The algorithm initially retrieved a total of 122 publications (Fig. 1). We excluded 72 manuscripts following the first step of the selection process, leading to $50 \mathrm{manu}-$ scripts (21 publications did not report on cancer; 51 publications did not report on a phase 1 trial). Following the second step of the review process, we subsequently excluded one manuscript specifically focusing on methodological issues, and 12 manuscripts due to absence of either an efficacy or toxicity endpoint or both. Based on the remaining 37 articles, 22 referred to the OBD and 15 did not refer to the OBD. We provide below a description of these two subgroups of manuscripts.

\section{Characteristics of the trials}

Characteristics of the selected trials are described in Table 1. The 37 trials were either phase I $(78.4 \%)$ or phase I/II trials (21.6\%). The 22 manuscripts reporting on OBD were more frequently phase I trials $(n=16$; $72.7 \%)$ as well as the 15 manuscripts that did not report on OBD $(n=13 ; 86.7 \%)$. Most trials reported on solid tumors $(n=28,75.7 \%)$, half of which were related to multiple organs $(n=14 / 28,50.0 \%)$. The cancer site did not vary substantially between trials reporting on OBD and those that did not. In articles reporting on OBD, about half reported on a single molecule and half on a combination of therapies. On the other hand, most of the articles that did not report on OBD focused more often on a single molecule (80.0\%). In articles reporting on OBD, the primary objective was either the MTD (31.8\%), the OBD $(50.0 \%)$ or both $(18.2 \%)$. Primary endpoint was the assessment of DLT (31.8\%), or a combination of endpoints involving DLT assessment and either PK, biological or clinical response (59.0\%). In articles that did not report on OBD, MTD was the primary objective for all manuscripts, and primary endpoint was DLT assessment. More than $80 \%$ of the articles mentioned a dose escalation design based on an algorithmic method $(3+3$, sequential cohorts or modified Fibonacci) associated with an observation period for DLT during the first cycle of treatment.

\section{Endpoints and methods considered in trials reporting on OBD}

The detailed characteristics of the 22 trials reporting the OBD are described in Table 2 . Trials focused mainly on targeted therapies $(n=12 / 22 ; 54.5 \%)$ and immunotherapies $(n=4 / 22 ; 18.2 \%)$. OBD was traditionally defined as the smallest dose maximizing an efficacy criterion such as biological activity. Efficacy was usually defined based on a biological target when OBD was the primary

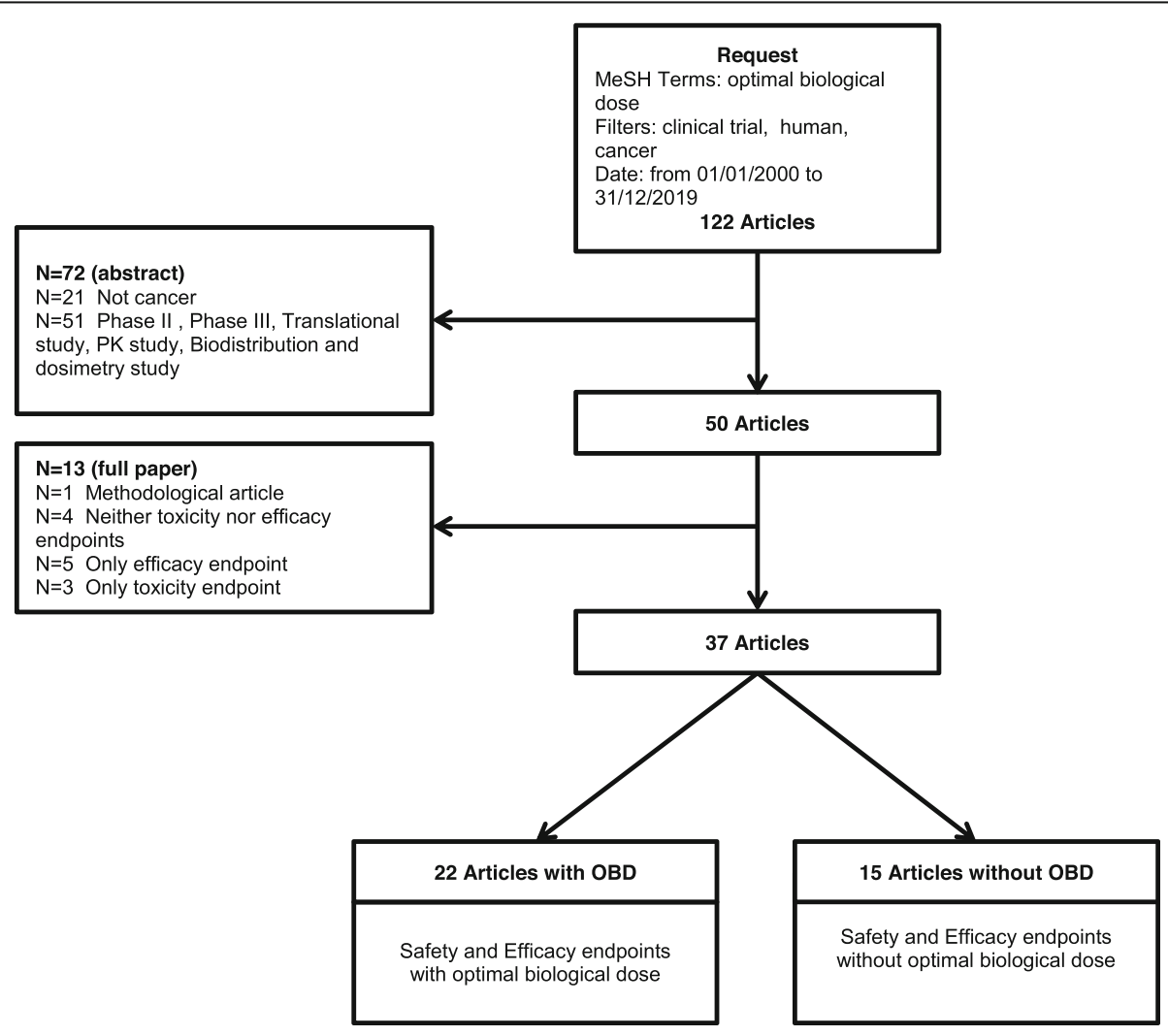

Fig. 1 Study selection 
Table 1 Characteristics of studies included in review

Trial
Phase I
Phase I/II
Location
Hematologic cancer
Solid tumor
Melanoma
Solid tumor + Melanoma
Solid tumor + Hematologic cancer

\section{Article with OBD}

$N=22$

Therapeutic schedule

Molecule

Association of molecules

Principal objective

MTD

$\mathrm{OBD}$

OBD/MTD

Primary endpoint

DLT

$\mathrm{DLT}+(\mathrm{PK}+$ clinical response)

$\mathrm{DLT}+$ biological target

$\mathrm{DLT}+$ clinical response

Biological target

Toxicity + biological target

\section{Observation period}

Not defined

First cycle

Other

Missing

\section{Dose-escalation method}

$$
3+3
$$

Modified fibonacci

CRM

Consecutive / Sequential cohorts

Other

DRP2

No

Yes

Secondary objective

OBD

Other

Secondary endpoint associated with OBD

$\mathrm{DLT}+$ biological target

DLT + clinical response

\section{6}

7

1

16

6

2

17

3

0

72.7

27.3

9.1

77.3

13.6

0

11

11

50.0

50.0

31.8

50.0

18.2

31.8

4.5

36.4

13.6

9.1

4.5

25.0

55.0

20.0

5

11

27.3

9.1

9.1

40.9

13.6

72.7

27.3

31.8

68.2

14.3

57.1

15

Article without OBD
$N=15 \quad \%$

Total

N=37 $\%$

86.7

29

78.4

13.3

8

21.6

13.3

73.3

6.7

6.7

80.0

20.0

100

4

10.8

75.7

6.7

28

8.1

2.7

2.7

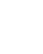

80.0
20.0

62.2

$\%$
78.4
21.6

10.8
75.7
8.1
2.7
2.7

62.2
37.8

59.5
29.7

3

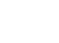

59.5

0

0

100

59.5

2.7

21.6

8.1

5.4

2.7

13.3

20.0

86.7

68.6

11.4

0

46.7

35.1

20.0

13.5

3

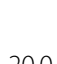

5.4

20.0

32.4

13.3

13.5

0

15

100

66.7

70.3

33.3

29.7

5

7

18.9

81.1
0
0
0.8

59.5
2.7
21.6
1
5.4
7

32.4

0

3.5


Table 1 Characteristics of studies included in review (Continued)

\begin{tabular}{|c|c|c|c|c|c|c|}
\hline & \multicolumn{2}{|c|}{ Article with OBD } & \multicolumn{2}{|c|}{ Article without OBD } & \multicolumn{2}{|l|}{ Total } \\
\hline & $N=22$ & $\%$ & $N=15$ & $\%$ & $N=37$ & $\%$ \\
\hline Toxicity + biological target & 2 & 28.6 & 0 & & 2 & 28.6 \\
\hline \multicolumn{7}{|l|}{ Secondary endpoint: PK/PD } \\
\hline No & 10 & 45.5 & 3 & 20.0 & 13 & 35.1 \\
\hline PK & 4 & 18.2 & 5 & 33.3 & 9 & 24.3 \\
\hline PD & 0 & & 1 & 6.7 & 1 & 2.7 \\
\hline PK/PD & 8 & 36.4 & 6 & 40.0 & 14 & 37.8 \\
\hline \multicolumn{7}{|c|}{ Secondary endpoint: Clinical response } \\
\hline No & 6 & 27.3 & 1 & 6.7 & 7 & 18.9 \\
\hline Yes & 16 & 72.7 & 14 & 93.3 & 30 & 81.1 \\
\hline \multicolumn{7}{|c|}{ Secondary endpoint: Immune response } \\
\hline No & 18 & 81.8 & 13 & 86.7 & 31 & 83.8 \\
\hline Yes & 4 & 18.2 & 2 & 13.3 & 6 & 16.2 \\
\hline \multicolumn{7}{|c|}{ Secondary endpoint: Survival outcome } \\
\hline No & 13 & 59.1 & 10 & 66.7 & 23 & 62.2 \\
\hline Yes & 9 & 40.9 & 5 & 33.3 & 14 & 37.8 \\
\hline
\end{tabular}

objective ( $n=11$ out of $15,73.3 \%$ ) as well as secondary objective ( $n=3$ out of $7,42.9 \%$ ). Biological endpoints included biological response (e.g. variation of biomarkers such as cells, proteins, microvessel density), immune cells count (cytokines, lymphocytes) for immunotherapies, or biological cell count (blood, urine) for targeted therapies. Few articles combining immunotherapy with biological agents and clearly mentioning the OBD have been identified $(n=3)$. For those particular cases, OBD was assessed using conventional DLT for safety and IR for efficacy. IR was specifically defined according to the studied molecule. The objective was to find among the safest doses the one with the highest immunogenicity $[16,36]$.

PK and PD measurements were considered as secondary objectives for the majority of trials $(n=12 ; 54.5 \%)$. PK studies included determination of plasma concentration profiles, distribution and clearance of the agent. The clinical response was most often evaluated as per RECIST criteria [37]: 4 (18.1\%) for primary endpoint and $16(72.7 \%)$ as secondary endpoint. With regards to survival outcomes, overall and progression free survivals were usually reported ( $n=9,40.9 \%)$.

Most trials relied on a dose-escalation design based on a single toxicity endpoint $(n=20,90.9 \%)$. In such case, DLT was the endpoint used to assess safety of the dose $(n=18,81.8 \%)$, otherwise a descriptive analysis of the reported events was provided $(\mathrm{n}=2,9.1 \%)$. Most trials $(n=19,86.4 \%)$ considered a binary toxicity endpoint defined in terms of the proportion of patients who experienced a dose-limiting toxicity (DLT; yes/no), based on protocol-specific adverse event definitions. Only two articles relied on an adaptive design (Bayesian CRM) which investigated a combination of multiple agents.

\section{Discussion}

We provided an overview of current evidence of phase 1 cancer trials relying on OBD. For those trials specifically reporting the OBD, OBD was considered either as a primary or secondary objective and usually associated with toxicity and efficacy endpoints in order to characterize toxicity with preliminary evidence of efficacy.

The toxicity endpoint was usually defined as a binary variable indicating the presence of DLT during the first cycle of treatment. In the retrieved articles, neither the cumulative toxicity nor DLT beyond the first treatment cycle were considered. Although the definition of efficacy depends on the mechanism of action of the molecule investigated, this review highlights that this definition was heterogeneous and not precisely reported. When the dose-efficacy relationship is non-monotonic, efficacy should be considered. This is particularly relevant for immunotherapy and targeted therapies, where efficacy usually reaches a plateau beyond a given dose. This is typically not the case for cytotoxic agents, for which most designs traditionally assume a monotonic dose-efficacy relationship, since it is expected that increased dose will lead to increased efficacy.

This review also highlights that the term OBD may be misused. Indeed, only two-thirds of manuscripts reporting on OBD actually considered it as a primary objective. For the other third, MTD was the primary objective and dose escalation relied only on the incidence of DLT and 


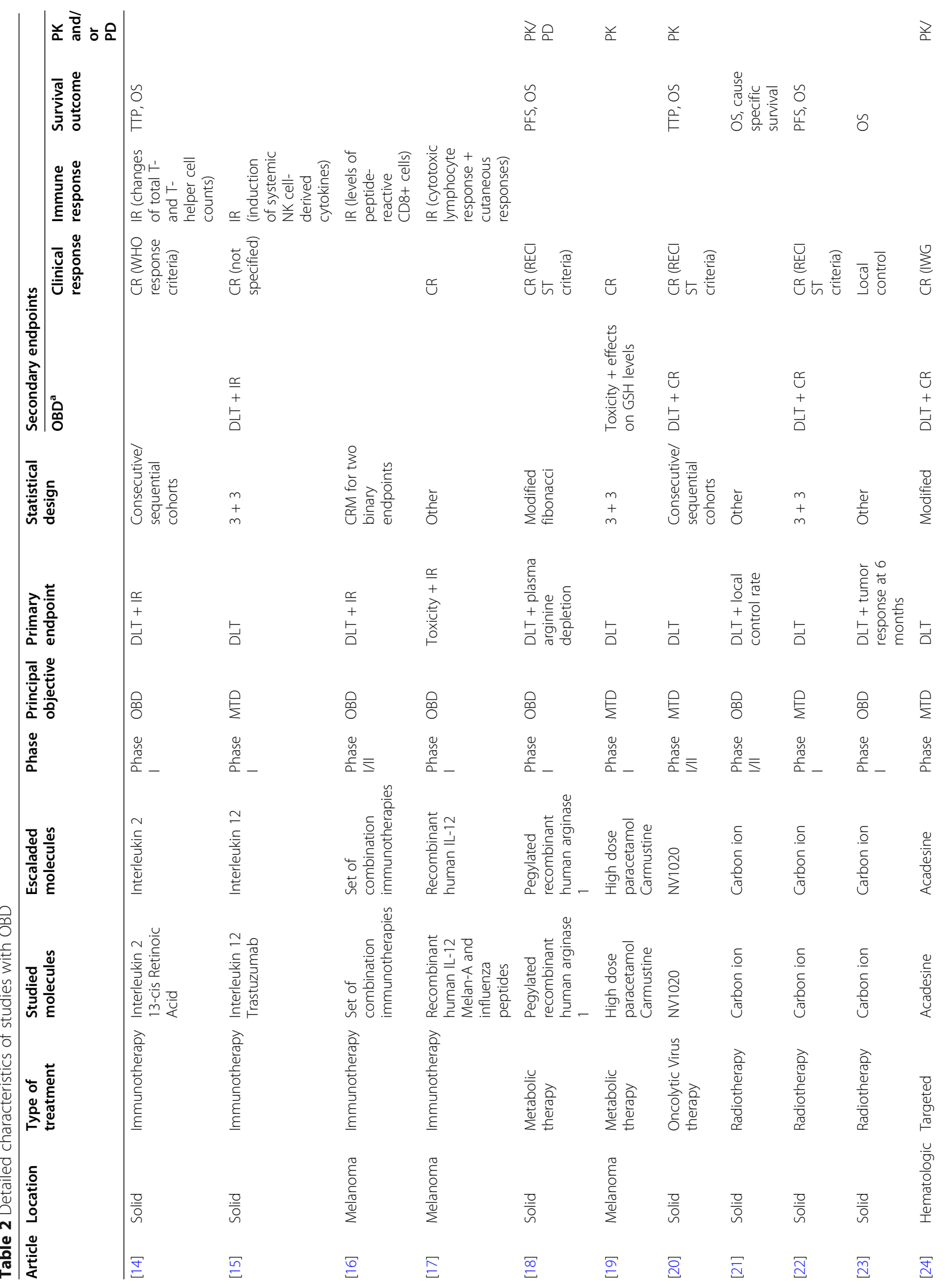




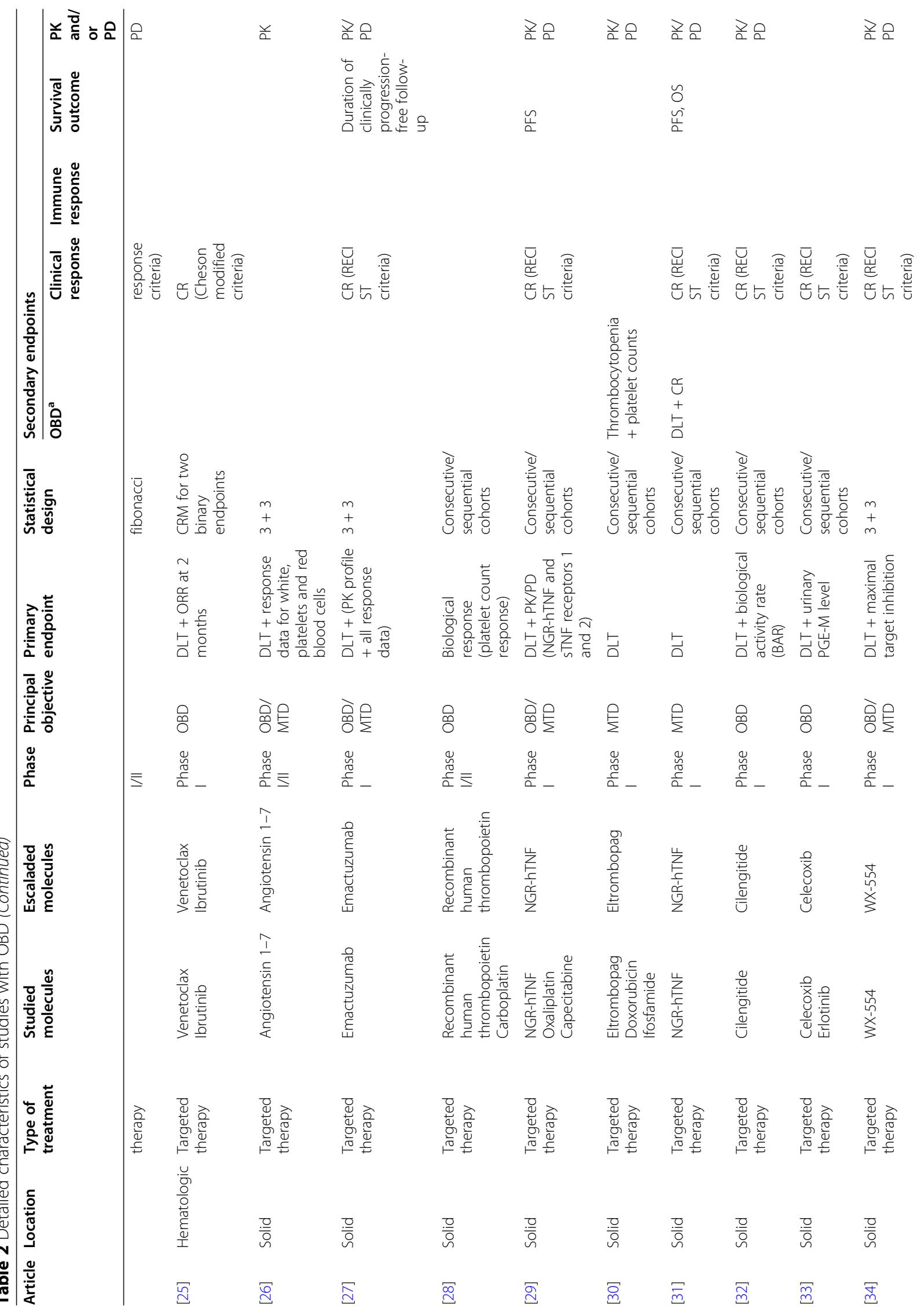




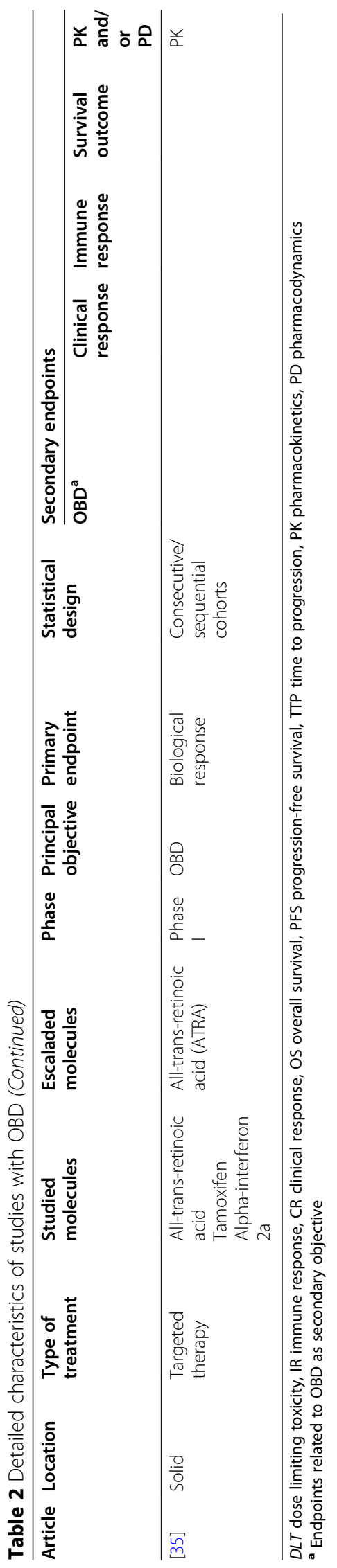


did not consider any efficacy data. Such approach may be appropriate to estimate the MTD but will not lead to the assessment of the OBD. In addition, trials targeting OBD as the primary objective should consider both toxicity and efficacy to proceed with dose escalation, which was clearly not the case for most trials as only two proceeded as such.

As a general recommendation, MTD should remain the primary objective in phase 1 trials investigating cytotoxic agents, while efficacy may be assessed as a secondary objective. On the other hand, phase 1 trials for immunotherapeutic and targeted agents should focus on OBD as the primary objective, and should thus jointly account for efficacy and toxicity when proceeding with dose escalation. In this specific setting, two approaches for dose escalation are promising, including designs relying on co-primary endpoints to jointly assess efficacy and toxicity, as well as designs accounting for efficacy only [36]. In this context, different methods exist but they are still underused in practice. These include extensions of the standard CRM in two directions for the modeling of both toxicity and efficacy in a phase I setting. In such extensions, one might consider preserving the bivariate structure of outcomes through a joint modeling of toxicity and efficacy [7]. On the other hand, it is possible to rely on a bivariate distribution for toxicity and efficacy defined either using a binary endpoint (e.g. progression) [38] or a continuous biomarker $[8,9]$. More recently, the joint modeling of longitudinal continuous biomarker activity measurements and time to first dose limiting toxicity has also been considered [39]. Finally, incorporating PK, PD or functional imaging as part of dose escalation has also been considered. Up to date, such designs however might be resource intensive which may have limited their application in phase I trials [40]. All these designs rely on the careful collection of all required safety and efficacy parameters, such as clinical and biological parameters.

\section{Conclusions}

OBD should be a primary objective for the assessment of the RP2D as part of targeted therapy or immunotherapy phase I trials in oncology and the statistical methods have to be adapted accordingly.

In the modern era of immunotherapy and targeted treatments, the concept of OBD has become particularly relevant in cancer phase I trials. As such, both toxicity and efficacy should be accounted for in the primary objective of such trials. Phase 1 designs should be adapted accordingly in order to account for both endpoints when proceeding with dose-escalation.

\section{Supplementary Information}

The online version contains supplementary material available at https://doi. org/10.1186/s12885-021-07782-z.

Additional file 1 Supplementary material 1. References of the 22 reviewed articles "With OBD". Supplementary material 2. References of the 15 reviewed articles "Without OBD". Supplementary material $\mathbf{3}$ Prisma Checklist.

\section{Abbreviations}

AUC: Area under the curve; CRM: Continual reassessment method; DLT: Dose limiting toxicity; CR : Clinical response; IR: Immune response; MTD: Maximum tolerated dose; OBD: Optimal biological dose; OS : Overall survival;

PFS: Progression-free survival; PD: Pharmacodynamics; PK: Pharmacokinetics; RP2D: Recommended phase 2 dose; TTP: Time to progression

\section{Acknowledgements}

NA

Authors' contributions

Study design: CB, CM, DT; Data acquisition: JF, DD; Statistical analysis: JF, DD; Manuscript preparation: all authors; all authors read and approved the manuscript.

\section{Funding}

The presented research was supported by a grant of the French National Cancer Institute (INCA-Grant n'SHS-ESP 2015-164).

(Note: The funding body had no other role in the design of the study and collection, analysis, and interpretation of data and in writing the manuscript.)

\section{Availability of data and materials}

NA

Ethics approval and consent to participate

NA

\section{Consent for publication}

NA

\section{Competing interests}

The authors have declared no conflicts of interest.

\section{Author details}

${ }^{1}$ Unité de Biométrie, Institut du Cancer Montpellier (ICM), Université de Montpellier, 208 rue des Apothicaire, 34298 Montpellier Cedex 5, France. IInserm ClC1401, Module Epidémiologie clinique, Institut Bergonié, Bordeaux, France. ${ }^{3}$ Institut Desbrest d'Epidémiologie et de Santé Publique, UMR Inserm - Université de Montpellier, Montpellier, France.

Received: 15 April 2020 Accepted: 1 January 2021

Published online: 13 January 2021

\section{References}

1. Le Tourneau C, Lee JJ, Siu LL. Dose escalation methods in phase I cancer clinical trials. J Natl Cancer Inst. 2009;101:708-20.

2. Eisenhauer EA, O'Dwyer PJ, Christian M, Humphrey JS. Phase I clinical trial design in cancer drug development. J Clin Oncol Off J Am Soc Clin Oncol. 2000;18:684-92.

3. Tosi D, Laghzali Y, Vinches M, Alexandre M, Homicsko K, Fasolo A, et al. Clinical development strategies and outcomes in first-in-human trials of monoclonal antibodies. J Clin Oncol. 2015;33:2158-65.

4. Paoletti X, Postel-Vinay S, Servois V, Doussau A, Ollivier L, Le Tourneau C. Dose finding methods for targeted agents: new perspectives. Bull Cancer (Paris). 2010;97:1485-95.

5. O'Quigley J, Pepe M, Fisher L. Continual reassessment method: a practical design for phase 1 clinical trials in cancer. Biometrics. 1990;46:33-48.

6. Piantadosi S, Liu G. Improved designs for dose escalation studies using pharmacokinetic measurements. Stat Med. 1996;15:1605-18. 
7. Braun TM. The bivariate continual reassessment method. Extending the CRM to phase I trials of two competing outcomes. Control Clin Trials. 2002; 23:240-56.

8. Bekele BN, Shen Y. A Bayesian approach to jointly modeling toxicity and biomarker expression in a phase I/II dose-finding trial. Biometrics. 2005;61: 343-54.

9. Dragalin $V$, Fedorov V. Adaptive designs for dose-finding based on efficacytoxicity response. J Stat Plan Inference. 2006;136:1800-23.

10. Dragalin V, Fedorov V, Wu Y. Adaptive designs for selecting drug combinations based on efficacy-toxicity response. J Stat Plan Inference. 2008;138:352-73.

11. Houede N, Thall PF, Nguyen H, Paoletti X, Kramar A. Utility-based optimization of combination therapy using ordinal toxicity and efficacy in phase I/II trials. Biometrics. 2010;66:532-40.

12. Moher D, Liberati A, Tetzlaff J, Altman DG. PRISMA group. Preferred reporting items for systematic reviews and meta-analyses: the PRISMA statement PLoS Med. 2009:6:e1000097.

13. PRISMA-P Group, Moher D, Shamseer L, Clarke M, Ghersi D, Liberati A, et al Preferred reporting items for systematic review and meta-analysis protocols (PRISMA-P) 2015 statement. Syst Rev. 2015;4:1.

14. Recchia F, De Filippis S, Rosselli M, Saggio G, Cesta A, Fumagalli L, et al. Phase 1B study of subcutaneously administered interleukin 2 in combination with 13-cis retinoic acid as maintenance therapy in advanced cancer. Clin Cancer Res Off J Am Assoc Cancer Res. 2001;7:1251-7.

15. Parihar R. A phase I study of interleukin 12 with Trastuzumab in patients with human epidermal growth factor Receptor-2-overexpressing malignancies: analysis of sustained interferon production in a subset of patients. Clin Cancer Res. 2004;10:5027-37.

16. Wages NA, Slingluff $C L$, Petroni GR. A phase $\mathrm{I} / \mathrm{II}$ adaptive design to determine the optimal treatment regimen from a set of combination immunotherapies in high-risk melanoma. Contemp Clin Trials. 2015;41: 172-9.

17. Cebon J, Jäger E, Shackleton MJ, Gibbs P, Davis ID, Hopkins W, et al. Two phase I studies of low dose recombinant human IL-12 with Melan-a and influenza peptides in subjects with advanced malignant melanoma. Cancer Immun. 2003:3:7

18. Yau T, Cheng PN, Chan P, Chan W, Chen L, Yuen J, et al. A phase 1 doseescalating study of pegylated recombinant human arginase 1 (peg-rhArg1) in patients with advanced hepatocellular carcinoma. Investig New Drugs. 2013;31:99-107.

19. Wolchok JD, Williams L, Pinto JT, Fleisher M, Krown SE, Hwu W-J, et al. Phase I trial of high dose paracetamol and carmustine in patients with metastatic melanoma. Melanoma Res. 2003;13:189-96.

20. Geevarghese SK, Geller DA, de Haan HA, Hörer M, Knoll AE, Mescheder A, et al. Phase I/II study of Oncolytic herpes simplex virus NV1020 in patients with extensively pretreated refractory colorectal Cancer metastatic to the liver. Hum Gene Ther. 2010;21:1119-28.

21. Miyamoto T, Yamamoto N, Nishimura H, Koto M, Tsujii H, Mizoe J, et al. Carbon ion radiotherapy for stage I non-small cell lung cancer. Radiother Oncol. 2003;66:127-40

22. Combs SE, Habermehl D, Ganten T, Schmidt J, Edler L, Burkholder I, et al. Phase i study evaluating the treatment of patients with hepatocellular carcinoma (HCC) with carbon ion radiotherapy: the PROMETHEUS-01 trial. BMC Cancer. 2011;11. https://doi.org/10.1186/1471-2407-11-67.

23. Makishima H, Yasuda S, Isozaki Y, Kasuya G, Okada N, Miyazaki M, et al. Single fraction carbon ion radiotherapy for colorectal cancer liver metastasis: A dose escalation study. Cancer Sci. 2018; cas.13872.

24. Van Den Neste E, Cazin B, Janssens A, González-Barca E, Terol MJ, Levy V, et al. Acadesine for patients with relapsed/refractory chronic lymphocytic leukemia (CLL): a multicenter phase I/II study. Cancer Chemother Pharmacol. 2013;71:581-91.

25. Wages NA, Portell CA, Williams ME, Conaway MR, Petroni GR Implementation of a model-based Design in a Phase lb Study of combined targeted agents. Clin Cancer Res. 2017:23:7158-64.

26. Rodgers KE, Oliver J, di Zerega GS. Phase $1 / / 1$ dose escalation study of angiotensin 1-7 $[A(1-7)]$ administered before and after chemotherapy in patients with newly diagnosed breast cancer. Cancer Chemother Pharmacol. 2006:57:559-68.

27. Cassier PA, Italiano A, Gomez-Roca CA, Le Tourneau C, Toulmonde M, Cannarile MA, et al. CSF1R inhibition with emactuzumab in locally advanced diffuse-type tenosynovial giant cell tumours of the soft tissue: a doseescalation and dose-expansion phase 1 study. Lancet Oncol. 2015;16:949-56.

28. Vadhan-Raj S, Verschraegen CF, Bueso-Ramos C, Broxmeyer HE, Kudelka AP, Freedman RS, et al. Recombinant human Thrombopoietin attenuates carboplatin-induced severe thrombocytopenia and the need for platelet transfusions in patients with gynecologic Cancer. Ann Intern Med. 2000; 132:364.

29. Mammoliti S, Andretta V, Bennicelli E, Caprioni F, Comandini D, Fornarini G, et al. Two doses of NGR-hTNF in combination with capecitabine plus oxaliplatin in colorectal cancer patients failing standard therapies. Ann Oncol. 2011:22:973-8.

30. Chawla SP, Staddon A, Hendifar A, Messam CA, Patwardhan R, Kamel YYM Results of a phase I dose escalation study of eltrombopag in patients with advanced soft tissue sarcoma receiving doxorubicin and ifosfamide. BMC Cancer. 2013;13. https://doi.org/10.1186/1471-2407-13-121.

31. Gregorc V, Citterio G, Vitali G, Spreafico A, Scifo P, Borri A, et al. Defining the optimal biological dose of NGR-hTNF, a selective vascular targeting agent, in advanced solid tumours. Eur J Cancer. 2010;46:198-206.

32. Hariharan S, Gustafson D, Holden S, McConkey D, Davis D, Morrow M, et al. Assessment of the biological and pharmacological effects of the av $\beta 3$ and av 35 integrin receptor antagonist, cilengitide (EMD 121974), in patients with advanced solid tumors. Ann Oncol. 2007:18:1400-7.

33. Reckamp KL. A phase I trial to determine the optimal biological dose of Celecoxib when combined with Erlotinib in advanced non-small cell lung Cancer. Clin Cancer Res. 2006;12:3381-8.

34. Jamieson D, Griffin MJ, Sludden J, Drew Y, Cresti N, Swales K, et al. A phase I pharmacokinetic and pharmacodynamic study of the oral mitogenactivated protein kinase kinase (MEK) inhibitor, WX-554, in patients with advanced solid tumours. Eur J Cancer. 2016;68:1-10.

35. Toma S, Raffo P, Nicolo G, Canavese G, Margallo E, Vecchio C, et al. Biological activity of all-trans-retinoic acid with and without tamoxifen and alpha-interferon 2a in breast cancer patients. Int J Oncol. 2000. https://doi. org/10.3892/ijo.17.5.991

36. Wages NA, Chiuzan C, Panageas KS. Design considerations for early-phase clinical trials of immune-oncology agents. J Immunother Cancer. 2018:6:81.

37. Eisenhauer EA, Therasse P, Bogaerts J, Schwartz LH, Sargent D, Ford R, et al. New response evaluation criteria in solid tumours: Revised RECIST guideline (version 1.1). Eur J Cancer. 2009;45:228-47.

38. Colin P, Delattre M, Minini P, Micallef S. An escalation for bivariate binary endpoints controlling the risk of Overtoxicity (EBE-CRO): managing efficacy and toxicity in early oncology clinical trials. J Biopharm Stat. 2017:27:1054-72.

39. Altzerinakou M-A, Paoletti $X$. An adaptive design for the identification of the optimal dose using joint modeling of continuous repeated biomarker measurements and time-to-toxicity in phase $\mathrm{I} / \mathrm{II}$ clinical trials in oncology. Stat Methods Med Res. 2019:096228021983773.

40. Cook N, Hansen AR, Siu LL, Abdul Razak AR. Early phase clinical trials to identify optimal dosing and safety. Mol Oncol. 2015:9:997-1007.

\section{Publisher's Note}

Springer Nature remains neutral with regard to jurisdictional claims in published maps and institutional affiliations.

Ready to submit your research? Choose BMC and benefit from:

- fast, convenient online submission

- thorough peer review by experienced researchers in your field

- rapid publication on acceptance

- support for research data, including large and complex data types

- gold Open Access which fosters wider collaboration and increased citations

- maximum visibility for your research: over $100 \mathrm{M}$ website views per year

At BMC, research is always in progress.

Learn more biomedcentral.com/submissions 